\title{
LEVELS OF BURNOUT SYNDROME IN CROATIAN CRITICAL CARE NURSES: A CROSS-SECTIONAL STUDY
}

\author{
Adriano Friganovici ${ }^{1,2,3}$ \& Polona Selič $\check{c}^{3}$ \\ ${ }^{I}$ Department of Anaesthesiology and Intensive Care, University Hospital Centre Zagreb, Zagreb, Croatia \\ ${ }^{2}$ Department of Nursing, University of Applied Health Sciences, Zagreb, Croatia \\ ${ }^{3}$ Department of Family Medicine, Faculty of Medicine, University of Ljubljana, Ljubljana, Slovenia
}

received: 5.11.2019;

revised: 23.11.2019;

accepted: 15.12 .2019

\section{SUMMARY}

Background: Burnout syndrome occurs in people who work in jobs which involve frequent and intense contact with people, especially healthcare workers. High dependency departments such as critical care units are very stressful environments, and this can lead to a greater incidence of burnout, especially of emotional exhaustion and poor personal accomplishment. Nurses are the largest group of healthcare workers, and so it is reasonable to expect they would have a high prevalence of burnout.

Subjects and methods: The aim of this study was to assess the prevalence of burnout in critical care nurses in Croatia and explore its association with demographic features. A cross-sectional study of 620 nurses was conducted in several university hospitals, using convenience sampling. The Maslach Burnout Inventory was administered, together with questions about the sociodemographic and work characteristics of the participants (age, gender, length of work in ICU, education, type of ICU).

Results: The majority of the sample were female nursing staff (87.7\%), aged 26-35 (38.9\%). The results showed that approximately every fifth nurse (22.1\%) expressed a high emotional exhaustion (EE), with lesser burden of a high depersonalisation (D) in $7.9 \%$, yet every third nurse (34.5\%) scored low on PA. Male nurses reported more depersonalisation ( $p=0.045$ ), yet neither EE nor the $P A$ dimensions differed by gender.

Conclusion: The results of this study concerning burnout are comparable to those of studies of other professions, but the results vary with regard to the sample and the working conditions of the countries.

Key words: burnout syndrome - incidence - nurses - critical care

$$
* * * * *
$$

\section{INTRODUCTION}

Burnout is known to be a psychological, work-related syndrome, and it develops as a result of long-term exposure to emotional and interpersonal stressors in the workplace (Selic et al. 2012). It manifests in negative self-esteem, a negative attitude towards work, and diminished interest in the clients/patients (Selic et al. 2012), and is characterised by emotional exhaustion, depersonalisation, and a lack of social accomplishment (Groene \& Jorgensen 2005, Friganovic et al. 2019). In nurses, burnout has been shown to be associated with adverse health outcomes, increased turnover, and decreased patient satisfaction (Florin \& Basham 2000).

Florin and Basham said that nurses who work in high-stress areas, such as critical care, report high levels of burnout (Florin \& Basham 2000). The leading causes of burnout in nurses are an imbalance between work requirements and preparation and fitness for the workplace, lack of control, insufficient performance recognition, and prolonged stress (Baker et al. 2004, Schaufeli $\&$ Buunk 2003). Hospital nurses have higher burnout scores compared to those working in other settings, due to moral distress, emotional and spiritual demands creating the perception of an excessive workload, and stresssors associated with physical and psychological environment-related characteristics (Florin \& Basham 2000).
Symptoms usually begin because of irregular adaptation to high stress demands (Friganovic et al. 2019). Due to burnout, nurses may develop addictions to alcohol or drugs, absenteeism, or diminished work capability (Stordeur et al. 2001), and have a cynical and negative view of themselves, a diminished sense of contentment (Miliken et al. 2007), and an increased risk of errors (Awa et al. 2010). The secondary consequences of burnout often include financial repercussions, including for the medical institution (Myhren et al. 2013).

The most typical stressors which lead to burnout have been identified: intra-group conflict, variation in workload, and job satisfaction (Elsaher et al. 2018). Inadequate patient safety and medical errors are certainly associated with the incidence of burnout (Alexandrova-Karamanova et al. 2016). Even critical care educators face some level of burnout associated with job satisfaction (Refaat Ahmed et al. 2018). Many authors suggest that improving work conditions may reduce burnout in critical care settings (Malaquin et al. 2017). Low personal accomplishment should be improved by supervisors valuing their team members better (Malaquin et al. 2017). Nurses who work in critical care settings without autonomy, and who are facing job instability and conflict, have a higher incidence of burnout than nurses who work in improved environments (Vahedimian Azimi et al. 2017). 
In this region, Macedonian and Croatian authors have conducted a study of burnout, work demands, stress, coping, and team working in hospital nurses, but no studies focused on critical care have been carried out in Croatia (Mijakoski et al. 2015, Šimunović 1998, Mesar et al. 2015). Only one study using nurses as participants (Mesar et al. 2015) investigated the prevalence of stress in surgical ward nurses. This study identified the most intense stressors in two groups of nurses (Mesar et al. 2015). Significant stressors that are related to the organisation of work were work overload; poor organisation; requests to work overtime, up to 24 hours; two-shift work; and limited time for working with patients (Mesar et al. 2015). In Slovenia, Selič et al. conducted a cross-sectional study of Slovenian family medicine trainees, nearly every fifth $(18.3 \%)$ scored high in all three dimensions (Selic et al. 2012). There is insufficient understanding of job satisfaction as a crucial factor in the development of burnout, and a need for more detailed research (Selič et al. 2012). Prevention of burnout syndrome should be a priority for multiple stakeholders in the healthcare system, and all levels of management must be involved in order to reduce its prevalence and prevent its effects on nurses, healthcare workers and the overall system (Moss \& Kleinpell 2019).

The intention of nurses to leave their profession has become a challenge for the modern healthcare system, and should be prevented by systematic activities such as improving the work environment (Moss \& Kleinpell 2019). Considering that nurses play a key role in managing futile medical care, being aware of their experiences in this regard could be an initial operational step towards compiling useful caregiving and educational programmes in intensive care units (Friganovic et al. 2017). Nurse managers should adopt supportive approaches and different strategies to reduce the prevalence of burnout syndrome in nurses (Friganovic et al. 2017).

The aims of this study were to explore the levels of burnout in critical care nurses in Croatia and the associations between burnout syndrome, age and gender. A quantitative cross-sectional multi-centre study was conducted in five Croatian hospitals.

\section{SUBJECTS AND METHOD}

\section{Participants}

The target population was critical care nurses employed in the intensive care units (ICU) of several Croatian University Hospitals. The nurses were asked to participate voluntarily in the study. A convenience sample was used; data collection was carried out from April to September 2017, and a total of 620 participants were recruited (544 female $(87.7 \%)$ and 76 male $(12.3 \%))$. The study was approved by the Ethical Committee of each of the hospitals included.
Table 1. Number and percentage of participants according to demographic features $(n=620)$

\begin{tabular}{|c|c|c|}
\hline & $\mathrm{n}$ & $\%$ \\
\hline \multicolumn{3}{|l|}{ Gender } \\
\hline Male & 76 & 12.3 \\
\hline Female & 544 & 87.7 \\
\hline \multicolumn{3}{|l|}{ Age in years } \\
\hline $18-25$ & 141 & 22.7 \\
\hline $26-35$ & 241 & 38.9 \\
\hline $36-45$ & 161 & 26.0 \\
\hline$>45$ & 77 & 12.4 \\
\hline \multicolumn{3}{|l|}{ Education } \\
\hline Vocational school & 318 & 51.3 \\
\hline Bachelor degree & 248 & 40.0 \\
\hline Master's degree & 54 & 8.7 \\
\hline \multicolumn{3}{|l|}{ Marital status } \\
\hline Single & 279 & 45.0 \\
\hline Married & 316 & 51.0 \\
\hline Divorced or widowed & 25 & 4.0 \\
\hline \multicolumn{3}{|l|}{ Work experience in years } \\
\hline$<5$ & 244 & 39.4 \\
\hline $5-10$ & 99 & 16.0 \\
\hline $11-15$ & 90 & 14.5 \\
\hline $16-20$ & 70 & 11.3 \\
\hline$>20$ & 117 & 18.9 \\
\hline \multicolumn{3}{|l|}{ Work department } \\
\hline Cardiac surgical ICU & 80 & 12.9 \\
\hline Neurosurgical ICU & 58 & 9.4 \\
\hline Paediatric and neonatal ICU & 73 & 11.8 \\
\hline Medical ICU & 80 & 12.9 \\
\hline Surgical ICU & 225 & 36.3 \\
\hline Coronary ICU & 72 & 11.6 \\
\hline Neurological ICU & 32 & 5.2 \\
\hline \multicolumn{3}{|l|}{ Organisation } \\
\hline University Hospital Centre Zagreb & 321 & 51.8 \\
\hline $\begin{array}{l}\text { University Hospital Centre Sestre } \\
\text { milosrdnice }\end{array}$ & 153 & 24.7 \\
\hline University Hospital Sveti Duh & 50 & 8.1 \\
\hline University Hospital Merkur & 34 & 5.5 \\
\hline University Hospital Dubrava & 62 & 10.0 \\
\hline
\end{tabular}

\section{Instruments}

Several demographic characteristics were collected, i.e. age, gender, education, duration of work in the ICU, marital status, and type of ICU. The Maslach Burnout Inventory (MBI) was designed to assess the three components of burnout syndrome: emotional exhaustion (EE), depersonalisation (DP), and reduced personal accomplishment (PA) (Maslach 1977). There are 22 items which are divided into three subscales. EE and DP are negative scales, so higher scores represent greater emotional exhaustion and depersonalisation. The PA score is a positive scale and a higher score represents higher PA. The Maslach Burnout Inventory (MBI) showed good Cronbach's alpha internal consistency, ranging from 0.74 to 0.90 for each subscale (Serec et al. 2012). 


\section{Statistics}

Descriptive statistics were used to analyse the main characteristics of the sample. The bivariate association between gender and burnout was examined using the chi-square test. Demographic features were selected as independent variables in the regression modelling, with burnout being the dependant variable.

Following Hsieh, the total of 620 participants was calculated to have more than $95 \%$ power to detect a significant association for logistic regression (using an alpha of 0.05 , a medium odds ratio of about 2.5 to 1 , and a variance inflation factor of 1.28) (Hsieh 1989, Resenthal 1996). The variance inflation factor, which depends on the squared multiple correlation coefficients $\left(\mathrm{R}^{2}\right)$ relating a specific predictor of interest to the remaining predictors, was calculated according to the instructions in Hsieh et al. (2003). Our study design did not involve one specific predictor of interest, so we calculated $\mathrm{R}^{2}$ for each predictor that was applied in the model and used the maximum value obtained. Since all the predictors were categorical variables, we applied logistic regression for Nagelkerke $\mathrm{R}^{2}$. Data analysis was carried out using IBM SPSS Statistics for Windows (version 22.0). Significance was set at $\mathrm{p}<0.05$.

\section{RESULTS}

Table 1 shows that the majority of the sample were female nursing staff (87.7\%) aged 26-35 (38.9\%). Most nursing staff had less than 5 years work experience (39.4\%); their primary work department was general surgery ICU (36.3\%); and most were located in University Hospital Centre Zagreb (51.8\%). The mean age was $33.5 \pm 9.5$ years, ranging from 19 to 62 years; work experience was SD 10.3 \pm 7.7 years, ranging from 1 to 38 years. According to the MBI dimension scores in Table 2 , the nursing staff expressed a high EE at $22.1 \%$, a high
DP at $7.9 \%$ and a low PA at $34.5 \%$. Men reported a higher percentage of medium and high DP in comparison to women $(\mathrm{p}=0.045)$, which is presented in Table 3 . The EE and PA levels did not differ by gender (Table 3). Table 4 shows the logistic regression modelling and the association between demographic features and dimensions of the MBI. The findings do not show any significant differences between $\mathrm{EE}$ and demographic features. Male gender $(\mathrm{OR}=2.03,95 \% \mathrm{CI}=1.20-3.42$, $\mathrm{p}=0.008$ ) was associated with higher depersonalisation. Master's degree education $(\mathrm{OR}=1.40,95 \% \mathrm{CI}=1.01-1.96$, $\mathrm{p}=0.045)$ and Bachelor degree education $(\mathrm{OR}=2.39$, $95 \% \mathrm{CI}=1.34-4.26, \mathrm{p}=0.003$ ) were associated with higher personal accomplishment. The range 5-10 years of work experience $(\mathrm{OR}=0.56,95 \% \mathrm{CI}=0.33-0.95, \mathrm{p}=0.032)$ was associated with lower personal accomplishment.

\section{DISCUSSION}

Critical care nurses in Croatia showed a high level of $\mathrm{EE}$ at $22.1 \%$, a high level of DP at $7.9 \%$ and a low PA at $34.5 \%$. Personal accomplishment was expressed as the most problematic dimension of the MBI. The results of a study Pejuskovic et al. conducted on physicians in Croatia also showed a high level of EE in $22.5 \%$, DP in $5.01 \%$ and PA in $37.3 \%$ in the overall sample, which correlates with the results of our study, but the authors did not present differences between gender and other demographic features (Pejuskovic et al. 2011). A Croatian group of authors also conducted a study of physicians and presented the results with a high score of EE in $42.4 \%$, DP in $16.0 \%$ and $\mathrm{PA}$ in $15.2 \%$; this difference is almost double our results (Ožvačić-Adžić et al. 2013). A comparison of these results is not highly relevant because of the different working groups and environmental settings, but it is certainly important for understanding the prevalence of burnout in healthcare workers (Ožvačić-Adžić et al. 2013).

Table 2. MBI dimensions by scoring level arrangement

\begin{tabular}{lccc}
\hline Scoring level & EE - Emotional exhaustion - n (\%) & DP - Depersonalisation - n (\%) & PA - Personal accomplishment n (\%) \\
\hline Low & $285(46.0)$ & $420(67.7)$ & $214(34.5)$ \\
Medium & $198(31.9)$ & $151(24.4)$ & $210(33.9)$ \\
High & $137(22.1)$ & $49(7.9)$ & $196(31.6)$ \\
\hline
\end{tabular}

Table 3. Burnout (MBI) subscales and total score according to gender

\begin{tabular}{lcccc}
\hline & Total - $\mathrm{n}=620(\%)$ & Male $-\mathrm{n}=76(\%)$ & Female $-\mathrm{n}=544(\%)$ & $\mathrm{p}^{*}$ \\
\hline EE - Emotional exhaustion & & & & 0.614 \\
$\quad$ Low & $285(46.0)$ & $31(40.8)$ & $254(46.7)$ & \\
Medium & $198(31.9)$ & $26(34.2)$ & $172(31.6)$ & \\
High & $137(22.1)$ & $19(25.0)$ & $118(21.7)$ & 0.045 \\
DP - Depersonalization & & & & \\
$\quad$ Low & $420(67.7)$ & $42(55.3)$ & $378(69.5)$ & \\
Medium & $151(24.4)$ & $26(34.2)$ & $125(23.0)$ & \\
High & $49(7.9)$ & $8(10.5)$ & $41(7.5)$ & 0.521 \\
PA - Personal accomplishment & & & & \\
Low & $214(34.5)$ & $25(32.9)$ & $189(34.7)$ & \\
Medium & $210(33.9)$ & $30(39.5)$ & $180(33.1)$ & \\
High & $196(31.6)$ & $21(27.6)$ & $175(32.2)$ & \\
\hline
\end{tabular}

* Chi-square test 


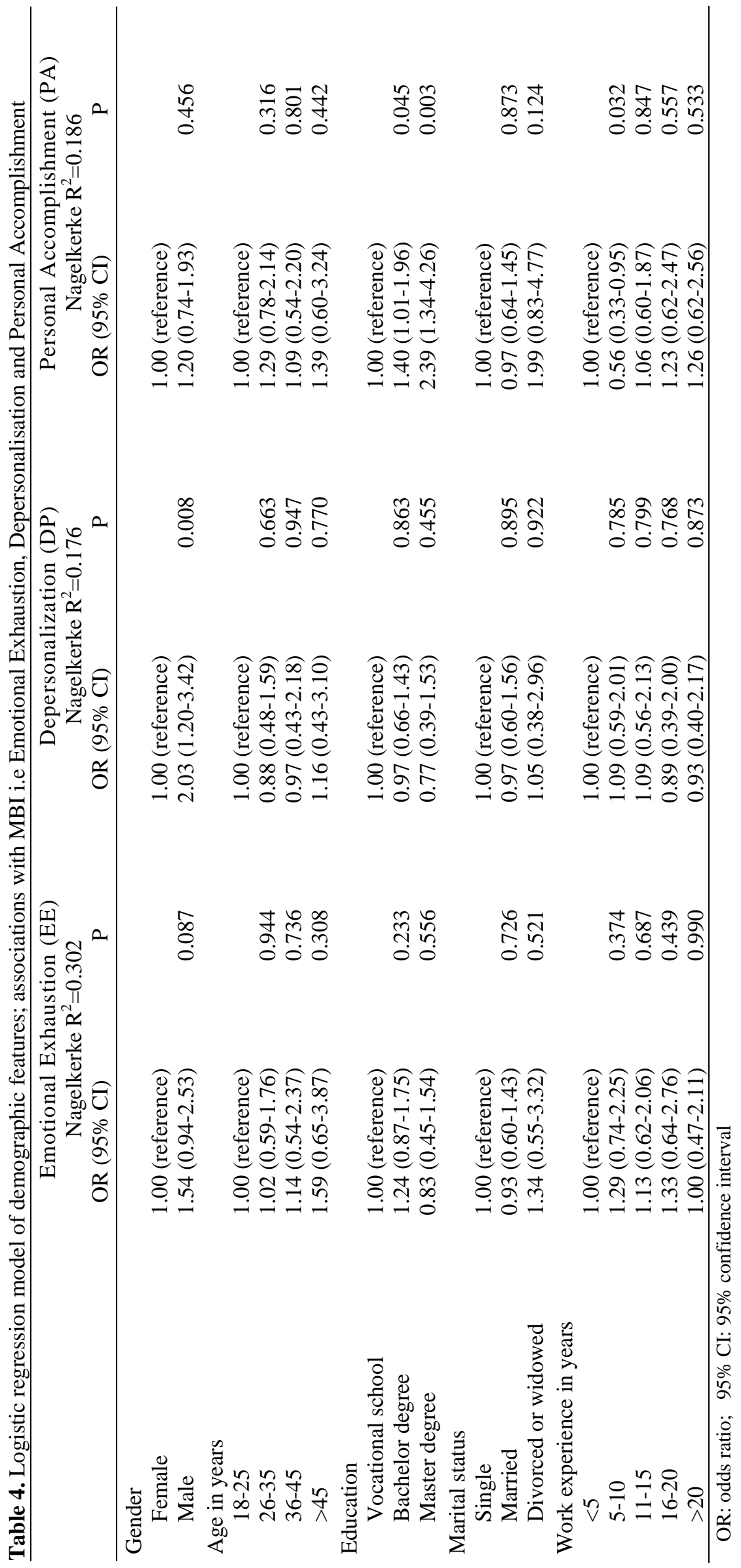


In Brazil a group of anaesthesiologists were tested with the MBI and the results they showed were similar to our results in the EE $(25.28 \%)$, but there was a difference in the DP (44.19\%) and PA (51.16\%) results (Barbosa et al. 2014). Sanchez-Moreno et al. compared nurses' results with that of social workers, and found differences in all three dimensions: EE in $12.2 \%$, DP in $26.5 \%$ and PA in 21.2\% (Sanchez-Moreno et al. 2015). A Macedonian group of researchers conducted a study in order to compare Croatian and Macedonian nurses; they found that Croatian nurses reported a higher level of DP than Macedonian nurses (Mijakovski et al 2011), which not correlate with the findings in our study. However, the authors did not distinguish any differences between clinical settings, and only tried to compare the size of the hospital (Mijakovski et al. 2011). A group of Slovenian authors reported a high level of EE (46\%) in a sample of family medicine trainees, which correlated with the findings of Ožvačić-Adžić et al., but they also found a high level of DP (42.4\%) and a low level of PA (15.2\%), which does not correlate with the reviewed studies (Selič et al. 2012, Ožvačić-Adžić et al. 2013). Selič et al. did not find any relationship between the MBI dimensions and gender, which was also shown in our study (Selič et al. 2012). Another study in Slovenia was conducted with soldiers, where the authors found a high level of EE in $16.3 \%$, a high level of DP in $8.64 \%$ and a low level of PA in $30.7 \%$, which relates to findings in our Croatian critical care nurses; the Slovenian authors also found a strong connection between MBI and job satisfaction (Selič et al. 2012).

A Saudi Arabian study conducted on a sample of 150 critical care nurses did not show any difference between the level of burnout and gender, just as we found in the nurses in Croatia (Alharbi et al. 2016). In this study there were also no differences between educational status and MBI dimension scores. Two Brazilian authors presented results on critical care nurses similar to the Croatian nurses, where they found a high level of EE in $22.0 \%$, a high level of DP in $9.1 \%$ and a low level of PA in $30.5 \%$ (Panunto \& Brito Guirardello 2013). Don Santos Alves et al. explored levels of emotional exhaustion in a sample of 267 paediatric nurses, and their results were almost the same as in the Croatian sample (EE in 21.5\%); the authors concluded that the nurses' autonomy and work environment correlated with emotional exhaustion (don Santos Alves et al. 2017). Results from a research study on 51 critical care nurses in Turkey (EE 14.68\%, DP $5.31 \%$, PA 19.19) also showed similar results to the Croatian sample in the dimensions of EE and DP, and there was a significant difference in PA (Denat et al. 2016). Nantsupawat et al. investigated the effects of burnout on quality of care on sample of 2084 nurses from Thailand (Nantsupawat et al. 2015). Compared to the Croatian results, their low PA score was almost the same (34.5\%), but the EE (32.2\%) and DP (17.8\%) showed significant differences, which may be related to the different clinical environment (Nantsupawat et al. 2015). This was one of the first studies of nurses which confirmed the relationship between the level of burnout and variables such as patient falls, medication errors, infections and poor quality of care (Nantsupawat et al. 2015). Montgomery et al. found similar results in two dimensions: EE in $20.85 \%$ and DP in $5.42 \%$ (Montgomery et al. 2015). A literature review did not find any studies with a relationship of gender or educational level and burnout.

\section{CONCLUSION}

The results of this study concerning burnout and dimensions of MBI in nurses are comparable to the results of studies of other professions, but the results vary with regard to the sample and the working conditions of the countries. However, for future research it would be interesting to compare different settings in clinical nursing: stressful and less stressful. The findings of the present study showed that male nurses have a significant increase in depersonalisation and prevention programmes should consider this fact. A higher level of nursing education showed higher results in personal accomplishment, which confirms the hypothesis that nurses' autonomy is a significant risk factor. The results also showed a relationship between low PA and work experience in the range 5-10 years. According to these findings it is clear that nurse education is a protective factor and nurses should be encouraged to obtain higher education. Preventive programmes should be directed towards male nurses and nurses with 5-10 years of work experience. Learning programmes related to burnout should be implemented in nurse education curricula.

\section{Acknowledgements:}

The authors acknowledge partial financial support from the Slovenian Research Agency's research core funding: "Research in the Field of Public Health No. P3-0339”, for the participation of Polona Selič.

\section{Conflict of interest : None to declare.}

\section{Contribution of individual authors:}

Adriano Friganović \& Polona Selič: study conception and design, data acquisition and selection, manuscript writing and revision.

\section{References}

1. Alharbi J, Wilson R, Woods C, Usher K: The factors influencing burnout and job satisfaction among critical care nurses: a study of Saudi critical care nurses. Journal of Nursing Management 2016; 24:708-17. doi:10.1111/jonm.12386

2. Alexandrova-Karamanova A, Todorova I, Montgomery A, Panagopoulou E, Costa P, Baban A, Davas A, Milosevic M, Mijakoski D: Burnout and health behaviours in health professionals from seven European countries. International Archives of Occupational and Environmental Health 2016; 89:1059-75

3. Awa LW, Plaumann M, Walter Ulla: Burnout prevention: A review of intervention programs. Patient Education and Counselling 2010; 78:184-90 
4. Baker AB, Demerouti E, Verbeke W: Using the job demands - resources model to predict burnout and performance. Hum Resource Manage 2004; 43:83-104

5. Barbosa FT, Jardelino Eloi R, dos Santos LM, Acioly Leao $B$, de Lima FJC, de Sousa-Rodrigues CF: Correlation between weekly working time and burnout syndrome among anaesthesiologists of Maceio-Al. Rev Bras Anestesiol 2017; 67:115-21

6. Denat Y, Gokce S, Gungor H, Zencir C, Akgullu C: Relationship of anxiety and burnout with extrasystoles in critical care nurses in Turkey. Pak J Med Sci 2016; 32:196-200

7. Don Santos Alves DF, da Silva D, de Brito Guirardello E: Nursing practice environment, job outcomes and safety climate: a structural equation modelling analysis. Journal of Nursing Management 2017; 25:46-55

8. Elsaher NSM, Moustafa MSA, Aiad MW, Ramadan MIE: Job Stress and Burnout Syndrome among Critical Care Healthcare Workers. Alexandria Journal of Medicine 2018; 54:273-77

9. Florin D, Basham S: Evaluation of health promotion in clinical settings. In: Thorogood $M$, Coombes $Y$, (Eds). Evaluating health promotion. Practice and methods. Oxford: Oxford University Press 2000; 140-50

10. Friganović A, Kovačević I, Ilić B, Žulec M, Krikšić V, Grgas Bile C: Healthy settings in hospital - how to prevent burnout syndrome in nurses: literature review. Acta Clin Croat 2017; 56:292-8. doi:10.20471/acc.2017.56.02.13292

11. Friganović A, Selič P, Ilić B, Sedić B: Stress and burnout syndrome and their associations with coping and job satisfaction in critical care nurses: a literature review. Psychiatr Danub 2019; 31(Suppl 1):s21-s31

12. Groene O, Jorgensen SJ: Health promotion in hospitalsa strategy to improve quality in health care. European Journal of Public Health 2005; 15:6-8

13. Hsieh FY: Sample size tables for logistic regression. Stat Med 1989; 8:795-802

14. Hsieh FY, Lavori PW, Cohen HJ, Feussner JR: An overview of variance inflation factors for sample-size calculation. Eval Health Prof 2003; 26:239-57

15. Malaquin S, Mahjoub Y, Musi A, Zogheib, Salomon A, Guilbart M, Dupont H: Burnout syndrome in critical care team members: A monocentric cross-sectional survey. Anaesth Crit Care Pain Med 2017; 36:223-8

16. Maslach C, Jackson SE, Leiter MP: Maslach Burnout Inventory. Evaluating stress: A book of resources 1977; 3:191-218

17. Mesar M, Koščak V, Starčević A: Cases of stress and how to reduce it in nurses at a Department of Traumatology Single Centre Experience SG/NJ 2015; 20:44-6

18. Mijakoski D, Karadzina-Bislimovska, Milosevic M, Mustajebegović J, Stoleski S, Minov J: Differences in Burnout, Work Demands and Team Work Between Croatian and Macedonian Hospital Nurses. Cognition, Brain, Behaviour 2015; 19:179-200

19. Miliken T, Clements P, Tillman H: The impact of stress management on nurse productivity and retention. Nursing Economic 2007; 25:203-10

20. Moss M, Klenpell R: An Official Critical Care Societies
Collaborative Statement - Burnout Syndrome in Critical Care Healthcare Professionals Chest 2016; 150:17-26

21. Montgomery A, Spanu F, Baban A, Panagopoulou: Job demands, burnout, and engagement among nurses: A multilevel analysis of ORCAB data investigating the moderating effect of teamwork. Burnout Research 2015; 2:71-9

22. Myhren H, Ekeberg Ø, Stokland O: Job Satisfaction and Burnout among Intensive Care Unit Nurses and Physicians. Critical Care Research and Practice 2013; 1-6

23. Ožvačić-Adžić Z, Katić M, Kern J, Soler JK, Cerovečki V, Polašek O: Is burnout in family physicians in Croatia related to interpersonal quality of care? Arh Hig Rada Toksikol 2013; 64:255-64

24. Panunto MR, de Brito Guirardello E: Professional nursing practice: environment and emotional exhaustion among intensive care nurses. Rev Latino - Am Enfermagem 2013; 21:765-72

25. Pejuskovic B, Lenčić-Toševski D, Priebe S, Tošković O: Burnout syndrome among physicians - the role of personality dimensions and coping strategies. Psychiatr Danub 2011; 23:389-95

26. Refaat Ahmed F, El-Sayed G, El-Haliem A, Mohamed Mostafa A: Stress, burnout, and job satisfactionof critical care nurse educators. Journal of Nursing and Health Science 2018; 7:30-7

27. Rosenthal JA: Qualitative descriptors of strength of association and effect size. J Soc Serv Res 1996; 21:37-59

28. Sanchez-Moreno E, de La Fuente Roldan IN, GallardoPeralta LP, de Roda ABL: Burnout, Informal Social Support and Psychological Distress among Social Workers. British Journal of Social Work 2015; 45:2368-86

29. Schaufeli WB, Buunk BP: Burnout: an overview of 25 years of research and theorizing. In: Schabracq MJ, Winnubst JAM, Cooper CC, (Eds). The handbook of work and health psychology. 2nd ed., West Sussex, England. Wiley 2003; 383-429

30. Selič P, Petek D, Pesjak K: Healthy but are they Satisfied? Factors Associated with Job Satisfaction in Professional Soldiers of the Slovenian Armed Forces. Journal of Defence Management 2012; 2:2-8

31. Selic P, Stegne-Ignjatovic T, Klemenc-Ketis Z: Burnout among Slovenian family medicine trainees: A crosssectional study. Zdrav Vestn 2012; 81:218-24

32. Serec M, Bajec B, Petek D, Švab I, Selič P: A structural model of burnout syndrome coping behaviour and personality traits in professional soldiers of the Slovene armed forces. Zdrav Vestn 2012; 81:326-36

33. Stordeur S, D'hoore W, Vanderberghe C: Leadership, organizational stress, and emotional exhaustion among hospital nursing. Journal of Advanced Nursing 2001; 35:533-42

34. Šimunović V: Suočavanje adolescenata $i$ odraslih sa svakodnevnim stresnim situacijama (Adolescent and adult coping with everyday stressed situations). Druš Istraž Zagreb 1998; 3:317-38

35. Vahedian-Azimi A, Hajiesmaeili M, Kangasniemi M, Fornés-Vives J, Hunsucker RL, Rahimibashar F, Pourhoseingholi MA, Farrokhvar L, Miller AC: Effects of Stress on Critical Care Nurses: A National Cross-Sectional Study 2019; 34:311-22. doi:10.1177/0885066617696853

Correspondence:

Adriano Friganović, $R N, B s N, M s N$

Department of Anaesthesiology and Intensive Care,

University Hospital Centre Zagreb

Kišpatićeva 12, 10000 Zagreb, Croatia

E-mail:adriano@hdmsarist.hr 